\title{
A Site Investigation of the 13 Housing Zones of the Chinese Dam Resettlement
}

\author{
Hai Lu
}

Graduate Student, Graduate School of Science and Engineering, Waseda University, M.Eng., Japan

\begin{abstract}
Since 1949 China has built over 86,000 dams, which has resulted in the involuntary resettlement of up to $12,000,000$ people. The major issue concerning resettlement is housing as this most directly affects the quality of life of those who are resettled. China has achieved significant results in resettlement housing construction; however the complexities of this resettlement process has raised tough problems. For example, there are regional diversities, rural vs. urban resettlement housing diversities and housing construction diversities resulting from different resettlement procedures. Following site investigation of 13 resettlement-housing zones in China, the author of this paper attempts to offer a systematic, analytical evaluation of the resettlement housing policies and current housing conditions in Chinese dam resettlement.
\end{abstract}

Keywords: dam; resettlement; involuntary resettler; housing policies; housing construction

\section{The Research}

\subsection{Background}

With the dual intention of ensuring hydro energy supply and minimizing flood disaster, the Chinese government has, since 1949 built a considerable number of dams. Today, these dams turn out to be a double-edge sword; i.e., although capable of generating $24.5 \%$ of the gross energy supplies for China, by and large 86,000 dams have resulted in the resettlement of over 12 million involuntary resettlers, a huge number in the history of human resettlement.

During this dam-resettlement process, Chinese resettlers had to face the following problems, "(it is hard to reside, hard to work, hard to gain water and electric power, hard to travel and hard to access medical aid and education.)" And, among these the housing problem proved to be a key issue in terms of stabilizing the resettlers, both psychologically and materially. A drop in the quality of life due to an unsuccessful housing policy only intimidates the resettlers, causing them to revert to their origins, and increasing social instability.

By setting improvement of living conditions as the first priority, the Chinese government has been consistently fine-tuning its policy with particular sympathy given to resettler's a vulnerable social group - by raising public awareness concerning their quality of life in general including housing and development and so on.

\footnotetext{
*Contact Author: Hai Lu, Graduate Student,

Graduate School of Engineering, Waseda University, C-0811, Tokyo International Exchange Center

2-79 Aomi, Koto-ku, Tokyo 135-0064 JAPAN

Tel: +81-3-5520-6896 Fax: +81-3-3209-8944

e-mail: luhai@suou.waseda.jp

(Recieved May 10, 2004; accepted April 22, 2005)
}

Ever since the eighties Chinese resettlement policy has been focused on economic issues, while troubleshooting research concerning housing issues has been as good as nonexistent. Thus it goes, without emphasizing the importance of a thought out and systematic study on the housing issue for the resettlers.

\subsection{Research Objective and History}

With the data and documentary information obtained from the site investigation of the 13 resettlements in China, the author of this paper shall try to produce an analytical evaluation of the housing construction for dam-resettlement.

In March and April, 2003, the author visited the Bureau of Resettlement and Development, the Ministry of Water Resource of People's Republic of China, Tsinghua University, Hohai University and the National Library of China, where he carried out research on the theory of resettlement, and shared views with resettlement officers and housing architects. In general, the focus has been on housing in this Chinese damresettlement.

Seen historically housing issues regarding resettlement in terms of how and where to resettle may vary significantly. Also, houses constructed by resettlers themselves are found scattered over a wide resettlement area. It is therefore difficult to carry out accurate statistical research for the entire nation's resettlement housing program. Up till now even the Chinese Central government does not have detailed housing construction data for the nation's resettlement; should a thorough investigation regarding the resettler's genuine housing condition be necessary, a site inquiry is essential. In August and September, 2003, with the cooperation of the Bureau of Resettlement and Development, the author carried out site studies of 13 resettler's housing sites spread over five provinces - SHANXI, HENAN, HUBEI, ANHUI and ZHEJIANG (Fig.1). 


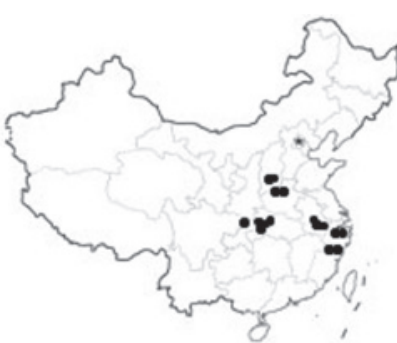

Fig.1. Dispersal of the Investigated Objects
Investigation samples for comparative study were selected from various time periods, built in different regions and carried out based on various resettlement models. It is hoped that with this sample research the paper will have some legitimacy. (Table 1 1-6, 9/A-M).

In China quite a lot of research on this resettlement issue has been published; yet the major concern has been on issues such as financial compensation, production recovery, and post resettlement patronage. Only two research papers slightly touch on the issue of resettler housing: Yuanfang LI's "Settlement for Low Income Resettlers and Urban Development" and Wanmin ZHAO's "The Research of Sustainable Human Settlement Construction in the Three Gorges Project of China." The former lays emphasis on the urbanization of resettlers from rural areas and the scale-control of cities. The latter draws attention to the building of new cities for those resettlers whose home towns were buried under water due to "The Three Gorges Project". In this paper the environmental capacity for the corresponding new cities is also examined. Both papers fail to encompass the field of resettlement housing, and construction policy, not to mention an analysis of housing design.

Such research has not yet been found in Japan.

In the West, two research papers were found which discuss resettlement housing problems: Shawn Steil' $\mathrm{s}$ "Problems in Rural Resettlement in Three-Gorges Project and Countermeasure" and Brooke McDonald' s "Reconstruction of a Village in China: Involuntary Resettlement and Culture." Yet both papers descriptive discussions regarding the resettlement housing issue in a resettlement village, is only partial without consistent and thorough analysis. Up till now there are none with respect to the Chinese Dam Resettlement housing, neither in its field of history, its policies, or its current status. The author of this paper will attempt to make the research significant in terms of protecting the rights and interests of the resettlers, upgrading their housing units in overall schemes and even produce detailed designs for improving future resettlement policy legislature.

\section{The Staging Aspect of the Resettlement Housing Construction}

Housing policy itself regarding dam-resettlement in China has never been independently documented; it is appendixed under the grand Dam-Resettlement Policy, and changes and fluctuates with the times.

Basing upon the historical and theoretical documents done form the field investigations and theoretical researches, dam-resettlement and its housing construction differ in three stages:

\section{1. $1949-1957$}

This stage encompasses the time-span from the birth of the People's Republic of China till the dawn of the so-called "Great Leap Forward (1958-60)."

1) "Construction First" was the slogan for the damresettlement policy in this period, stressing its contribution to the nation's best interests.

In the National Conference for Water Resources held in 1953 the administration made an announcement regarding dam-resettlement: "A dam or flood-reserved area must be built or developed on condition that the resettler population is kept to a minimum and that their living standard shall not be made worse then their original one. Moreover, the government shall offer the resettlers compensation during the resettlement process without offending the local populations interests, consolidating both the resettlers and the local populace with political education." In January of 1953 this conference also delivered the "Land Requisition Policy for National Development" which had been the principle for dam-resettlement at this stage.

These policies were set, however the then weak Chinese economy and construction capability could not fulfill the promises made in the policies for the resettlers, neither in quality nor in quantity. As a result, being carried out by the basic labor unit — Production Brigade - without proper governmental compensation in the rural areas, the housing construction resulted in low quality dwellings. Examples such as "clay houses" found in the Southern area, "kiln caves" in the Northwestern hilly slope area and "timber clay houses" in northern China (Photos 1, 2 and 3).

In the fifties the Chinese people in general were living in poverty. The resettlers, moving with very little personal belongings, were capable of adapting to their dwellings without difficulty. The Bureau of DamResettlement also managed to minimize problems in the process.

\section{$2.21957-1985$}

This was a chaotic time following the birth of the PRC. Suffering the impact of "The Great Leap Forward" and "The Great Proletarian Cultural Revolution,"2) the Bureau of Dam-Resettlement was thwarted by a lack of executive capability by which to carry out its duties.

During this time, due to ineffective management, resettlement housing was low in quality and thus resulted in the so-called "four none" - none consistent planning, none consistent scheme, none consistent construction, none consistent management.

Production of cement, steel, bricks and so on were low and most were used for politically motivated construction. The resettlement dwelling units were built largely depending on recycled materials (Table $111 / \mathrm{A}, \mathrm{B})$. Lacking both management and planning, these units were built by resettlers themselves and turned out to be low-quality structures without strength, endurance or shake-resistance. They were 
nothing but units for the most basic living conditions for resettlers. (Photos 4, 5 and 6)

The perfunctorily chosen sites for resettler housing and poor production further worsened their living conditions. Daily traveling distance between homes and production site, the farming of livestock and crops and the like, was distant; accessibility to electricity, water and child education were scarce. A considerable number of people were resettled to remote mountain ridge areas without even proper water supply and their post-resettlement lives, especially the hygienic conditions were rather bad (Table 1 18/A, B). Transportation was hard due to a lack of highway construction funds; even today connection with the outside world is still inconvenient (Table $121-23 / \mathrm{A}, \mathrm{B}$ ).

This investigation shows that the resettlement housing at that time had problems such as "undersize," insufficient living space, "aging," buildings constructed with recycled materials, "overcrowding" multi-generation shared accommodation and "unfair distribution," a unit shared by several families or a family occupying several units (Table 1 13-15/A, B).

Problems such as these are now taken into consideration by the Chinese Central Government.

\subsection{Since 1985}

A remarkable achievement of this stage was resumption of the Bureau of Resettlement in 1985. Following the "6th Plenary Session of the 11th CPC Central Committee" held by the Communist Party of China in June 1981, the economic system was converted to a market economy by which personal property is permitted and protected. This new economic system certainly affected resettlement policies.

The greatest benefit to arise from the new policy was a rise in compensation and contracted rather than compulsory resettlement. Housing policies were also updated in two ways.

The first was the so-called "Three-Originality" principles for housing construction set up to protect the interests of the resettlers; i.e., "Original Size", "Original Standard" and "Original Function".

Second one was the so-called "Five-uniform" Principles; that is, "unifying land distribution, a unifying scheme, unifying planning, unifying investigation and unifying management". Such policies were put into practice and strived for thoroughness, standing on firm ground during the resettlement process.

With these new housing policies the resettlers enjoy better quality housing units and higher hygienic facilities. Clay caves are history, brick or brick $+\mathrm{RC}$ units, with an average living area per person higher than the national standard ${ }^{3}$ ), were seen in resettlement areas. Construction materials and interior decorative materials were improved. Medicare, public and educational facilities were significantly improved (Table 1 14-20/C-M).

With the five-uniform principles being carried out, the Bureau of Resettlement demanded the so-called "typical designs" of the Design Department who presented in return at least three design proposals. These proposals were inspected by the Bureau of Resettlement and representatives from the resettlers; and the chosen proposal was constructed. This procedure is now commonly recognized and available nationwide. The significance here is that since then resettlement housing has begun to be standardized and schematized (Table 1 8/C-M) (Figs. 2 and 3).

\section{Chinese Resettlement Housing Construction: its Characteristics}

According to field investigation, resettlement housing construction in China, after 50 years of development, has made the following significant achievements:

1) Increased living area. The average living area perperson for a dwelling from the 50s to 70 s was either equal to or smaller than its pre-settlement counterpart. Yet, since the late $80 \mathrm{~s}$, resettlement-housing units have enjoyed a larger average living area. (Table 1 15/A-M)

2) Upgraded hygiene conditions. Since the late 80 s resettlement housing units have abandoned the traditional self-discharge toilets and been properly equipped with the two-urn-funnel toilets and flush toilet, which is a much more sanitary solution. (Table 1 18/A-M)

3) Improved construction. The much safer brick $+\mathrm{RC}$ construction rather than clay construction was commonly used. (Table 1 11/A-M)

4) Enforced construction management. Construction processes were placed under unified management from design to construction.

5) Improved infrastructure and appliances for housing units. In addition to the common housing units, the required schools, hospitals and traffic facilities were also taken into account (Table 1 19, 20, 22, 23/A-M).

Through investigation, the author has discovered several problems, which need to be tackled.

\subsection{Regional Diversity}

The evidence of regional diversity is obvious, as indicated in the site research. Housing units built in different resettlements during the same period differ greatly. The key issue in assessing housing unit quality is the average dwelling area per person. The highly developed and prosperous city of WENZHOU in ZHEJIANG province Eastern China for instance, has an average resettlement living area per person twice as much or even more than that of SHANXI and HENAN provinces in less economically developed Western China. Besides, construction techniques and decorative materials in the Eastern economic zone are definitely better than the Western equivalent (Table 1. 3, 10-23/ C-F, J-M) (Photos 7 and 8).

Before the eighties, since economic development in China was implemented in the style of the Socialist 
Table 1. Site Investigation Data ( "Before/After" is a Abbreviation of "Before the Resettlement / After the Resettlement")

\begin{tabular}{|c|c|c|c|c|c|c|c|}
\hline \multirow{3}{*}{\multicolumn{2}{|c|}{$\mathrm{X}^{\text {Investigated Objects }}$}} & $\mathrm{A}$ & $\mathrm{B}$ & $\mathrm{C}$ & $\mathrm{D}$ & E & $\mathrm{F}$ \\
\hline & & \multicolumn{2}{|c|}{ ANHUI Province } & \multicolumn{2}{|c|}{ SHANXI Province } & \multicolumn{2}{|c|}{ HENAN Province } \\
\hline & & $\begin{array}{l}\text { New YANG Village, } \\
\text { SHEXIAN, }\end{array}$ & $\begin{array}{c}\text { ZHANGTAN Village, } \\
\text { SHEXIAN, }\end{array}$ & $\begin{array}{l}\text { ZAILI Village, } \\
\text { HUANQU }\end{array}$ & $\begin{array}{l}\text { XIABO Village, } \\
\text { HUANQU }\end{array}$ & $\begin{array}{l}\text { MAYU Village, } \\
\text { WENXIAN }\end{array}$ & $\begin{array}{c}\text { CANGTOU Village, } \\
\text { WENXIAN }\end{array}$ \\
\hline 1 & Chief Administrative organization & \multicolumn{6}{|c|}{ Bureau of Resettlement and Development, Ministry of Water Resource of People's Republic of China } \\
\hline 2 & $\begin{array}{l}\text { Resettlement Under the Dam's } \\
\text { Jurisdiction/ } \\
\text { The Total Dam Resettler } \\
\text { Population }\end{array}$ & \multicolumn{2}{|c|}{$\begin{array}{l}\text { XIN'ANJIANG Dam/ } \\
306 \text { Thousand }\end{array}$} & \multicolumn{4}{|c|}{$\begin{array}{l}\text { XIAOLANGDI Dam, Yellow River/ } \\
200 \text { Thousand }\end{array}$} \\
\hline 3 & $\begin{array}{l}\text { Region for Investigated articles/ } \\
\text { Regional Economic Situation }\end{array}$ & \multicolumn{2}{|c|}{$\begin{array}{l}\text { Mid-eastern China/ } \\
\text { Underdeveloped }\end{array}$} & \multicolumn{2}{|c|}{$\begin{array}{l}\text { Midwestern China/ } \\
\text { Underdeveloped }\end{array}$} & \multicolumn{2}{|c|}{$\begin{array}{l}\text { Central China/ } \\
\text { Average }\end{array}$} \\
\hline 4 & $\begin{array}{c}\text { Resettlement Method/Resettlement } \\
\text { Distance }\end{array}$ & \multicolumn{2}{|c|}{$\begin{array}{c}\text { Local-Rear-Resettlement } \\
/ /-\end{array}$} & $\begin{array}{c}\text { Distance Resettlement } \\
\text { (Inside the Province) } \\
\text { /100KM }\end{array}$ & $\begin{array}{c}\text { Distance Resettlement } \\
\text { (Inside the Province) } \\
\text { /80KM }\end{array}$ & $\begin{array}{c}\text { Distance Resettlement } \\
\text { (Inside the Province) } \\
/ 210 \mathrm{KM}\end{array}$ & $\begin{array}{c}\text { Distance Resettlement } \\
\text { (Inside the Province) } \\
/ 110 \mathrm{KM}\end{array}$ \\
\hline 5 & $\begin{array}{l}\text { The year the housing was } \\
\text { constructed }\end{array}$ & 1958-66/3 Stage & $1958,74,78 / 3$ Stage & $1995-97$ & $1995-98$ & 1998.5.8 & 1998.8-99.4 \\
\hline 6 & $\begin{array}{l}\text { Resettler population in the } \\
\text { investigated object } \\
\text { (Unit / People) }\end{array}$ & $103 / 311$ & $460 / 1503$ & $205 / 788$ & $286 / 1072$ & $226 / 756$ & $668 / 2359$ \\
\hline 7 & $\begin{array}{l}\text { Urban } \bullet \text { Rural } \\
\text { (Before/After) }\end{array}$ & Rural/Rural & Rural/Rural & Rural/Rural & Rural/Rural & Rural/Rural & Rural/Rural \\
\hline 8 & $\begin{array}{l}\text { Typical Design: availability / } \\
\text { Scheme Change: Availability }\end{array}$ & $\mathrm{No} / \mathrm{No}$ & $\mathrm{No} / \mathrm{No}$ & Yes/Yes & Yes/Yes & Yes/Yes & Yes/Yes \\
\hline 9 & Housing Construction Method & Self Construction & Self Construction & Self Construction, & Self Construction, & Self Construction, & Self Construction, \\
\hline 10 & Housing Type Before/After & \multicolumn{2}{|c|}{ Independent housing/Independent housing } & \multicolumn{4}{|c|}{ Independent housing/Linear Housing ${ }^{6)(\text { Photol6, Fig6) }}$} \\
\hline 11 & $\begin{array}{l}\text { Structure Form } \\
\text { (Before/After) }\end{array}$ & $\begin{array}{l}\text { Clay, Brick } \\
\text { /Clay, Brick }\end{array}$ & $\begin{array}{l}\text { Clay, Brick } \\
\text { /Clay, Brick }\end{array}$ & $\begin{array}{l}\text { Clay, Kiln Cave } \\
\text { /Brick+RC }\end{array}$ & $\begin{array}{l}\text { Clay, Kiln Cave, } \\
\text { Brick/Brick+RC }\end{array}$ & $\begin{array}{l}\text { Clay, Brick } \\
\text { /Brick+RC }\end{array}$ & $\begin{array}{l}\text { Clay, Kiln Cave, } \\
\text { Brick/Brick+RC }\end{array}$ \\
\hline 12 & Storey Before/After & $1-2 / 1-2$ & $1-2 / 1-2$ & $1 / 2$ & $1 / 2$ & $1 / 1-3$ & $1 / 1-3$ \\
\hline 13 & $\begin{array}{c}\text { Number of generations living } \\
\text { together in one unit }\end{array}$ & $0-2$ & $0-3$ & $1-3$ & $1-3$ & $1-3$ & $1-3$ \\
\hline 14 & $\begin{array}{l}\text { Average housing site area } \\
\text { per person }\left(\mathrm{m}^{2}\right)\end{array}$ & 20 & 16 & 50 & 50 & 80 & 80 \\
\hline 15 & $\begin{array}{l}\text { Average dwelling housing area } \\
\text { per person Before/After }\left(\mathrm{m}^{2}\right)\end{array}$ & $30 / 25$ & $29 / 24$ & $20 / 30$ & $20 / 35$ & $25 / 26$ & $25 / 40$ \\
\hline 16 & Decorative materials for exterior & Whitewash & Whitewash & Whitewash, Tile & Whitewash, Tile & Whitewash, Tile & Whitewash, Tile \\
\hline 17 & $\begin{array}{l}\text { Interior decorative materials } \\
\text { (Floor/Wall) }\end{array}$ & Cement/Whitewash & Cement/Whitewash & $\begin{array}{l}\text { Cement, Tile } \\
\text { /Whitewash }\end{array}$ & $\begin{array}{l}\text { Cement, Tile } \\
\text { /Whitewash }\end{array}$ & $\begin{array}{c}\text { Cement, Tile } \\
\text { /Whitewash·Stucco }\end{array}$ & $\begin{array}{c}\text { Cement, Tile } \\
\text { /Whitewash, Stucco }\end{array}$ \\
\hline 18 & $\begin{array}{l}\text { Hygienic condition } \\
\text { (Toilet) (Before/After) }\end{array}$ & $\begin{array}{l}\text { Self-discharge/Self } \\
\text { discharge } 7 \text { (Photol7) }\end{array}$ & $\begin{array}{l}\text { Self-discharge Toilet/ } \\
\text { Self-discharge }\end{array}$ & \begin{tabular}{|c|}
$\begin{array}{c}\text { Self-discharge Toilet } \\
\text { (Two-Urn-Funnel } \\
\text { Toilet }^{8)(\text { Photol 18) }}\end{array}$ \\
\end{tabular} & $\begin{array}{c}\text { Self-discharge Toilet } \\
\text { /Two-Urn-Funnel } \\
\text { Toilet }\end{array}$ & $\begin{array}{c}\text { Self-discharge Toilet } \\
\text { /Two-Urn-Funnel } \\
\text { Toilet } \\
\end{array}$ & $\begin{array}{c}\text { Self-discharge Toilet } \\
\text { /Two-Urn-Funnel } \\
\text { Toilet } \\
\end{array}$ \\
\hline 19 & Public facilities & $\begin{array}{l}\text { Ancestral temple, } \\
\text { Grocery }\end{array}$ & $\begin{array}{l}\text { Ancestral temple, } \\
\text { Grocery }\end{array}$ & Grocery & $\begin{array}{l}\text { Open air Theater, } \\
\text { Grocery }\end{array}$ & Dispensary, Grocery & $\begin{array}{l}\text { Hospital, square, } \\
\text { recreation room, } \\
\text { Almshouse }\end{array}$ \\
\hline 20 & School & - & Grade school & $\begin{array}{l}\text { Kindergarten, } \\
\text { Grade school }\end{array}$ & $\begin{array}{l}\text { Kindergarten, } \\
\text { Grade school }\end{array}$ & Grade school & $\begin{array}{l}\text { Grade school, } \\
\text { High school }\end{array}$ \\
\hline 21 & $\begin{array}{l}\text { Distance between house and } \\
\text { production site }\end{array}$ & $2-6 \mathrm{Km}$ & $1-7 \mathrm{Km}$ & $0-3 \mathrm{Km}$ & $1-4 \mathrm{Km}$ & $1-3 \mathrm{Km}$ & $1-3 \mathrm{Km}$ \\
\hline 22 & $\begin{array}{l}\text { Road availability in } \\
\text { residential zone/Width }\end{array}$ & No & No & $\mathrm{Yes} / 5 \mathrm{~m}, 3.5 \mathrm{~m}$ & $\mathrm{Yes} / 5 \mathrm{~m}, 3.5 \mathrm{~m}$ & Yes $/ 6 \mathrm{~m}, 3.5 \mathrm{~m}$ & Yes $/ 10 \mathrm{~m}, 6 \mathrm{~m}, 3.5 \mathrm{~m}$ \\
\hline 23 & $\begin{array}{l}\text { The means for transportation } \\
\text { to the cities / convenience }\end{array}$ & $\begin{array}{l}\text { Bike, Motorcycle } \\
\text { /Inconvenient }\end{array}$ & $\begin{array}{l}\text { Bike, Motorcycle } \\
\text { /Inconvenient }\end{array}$ & $\begin{array}{l}\text { Bike, Motorcycle } \\
\text { /Inconvenient }\end{array}$ & $\begin{array}{l}\text { Bike, Motorcycle } \\
\text { /Inconvenient }\end{array}$ & $\begin{array}{l}\text { Bike, Motorcycle, } \\
\text { Farming-vehicle/ } \\
\text { convenient }\end{array}$ & $\begin{array}{l}\text { Bike, Motorcycle, } \\
\text { Farming-vehicle/ } \\
\text { convenient }\end{array}$ \\
\hline 24 & $\begin{array}{l}\text { Occupation changes } \\
\text { (Before/After) }\end{array}$ & Farming/Farming & Farming/Farming & $\begin{array}{c}\text { Farming/ } \\
\text { Farming, Trade }\end{array}$ & $\begin{array}{c}\text { Farming/ } \\
\text { Farming, Trade }\end{array}$ & $\begin{array}{c}\text { Farming/ } \\
\text { Farming, Trade }\end{array}$ & $\begin{array}{c}\text { Farming/ } \\
\text { Farming,Trade, } \\
\text { Packing industry }\end{array}$ \\
\hline 25 & $\begin{array}{c}\text { Housing design adjustment } \\
\text { in accordance with occupational } \\
\text { change }\end{array}$ & No & No & $\begin{array}{l}\text { Commercial Area } \\
\text { Reserved in the } \\
\text { Housing Units along } \\
\text { both Sides of the } \\
\text { Main Street }\end{array}$ & \begin{tabular}{|c|} 
Commercial Area \\
Reserved in the \\
Housing Units along \\
both Sides of the \\
Main Street
\end{tabular} & \begin{tabular}{|c|} 
Commercial Area \\
Reserved in the \\
Housing Units along \\
both Sides of the Main \\
Street
\end{tabular} & $\begin{array}{c}\text { Commercial Area } \\
\text { Reserved in the } \\
\text { Housing Units along } \\
\text { both Sides of the } \\
\text { Main Street }\end{array}$ \\
\hline & & A & B & $\mathrm{C}$ & $\mathrm{D}$ & E & F \\
\hline
\end{tabular}

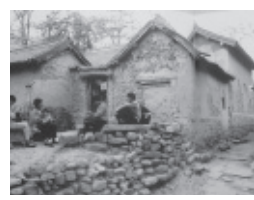

Photo 1. "Clay houses"

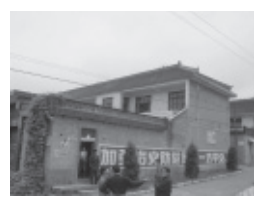

Photo 7. XIABO Village, (Western China)

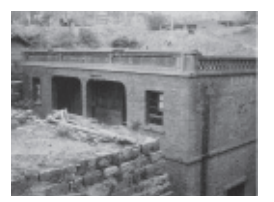

Photo 2. "Kiln caves"

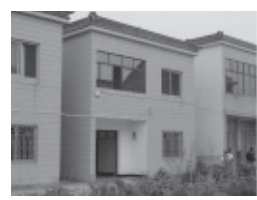

Photo 8. No.7 Group, QIJIAQIAO Photo 9. Rural Housing

Village, ZHEJIANG Province -ZIGUI, HUBEI Province (Eastern China)

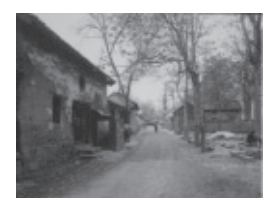

Photo 3. "Timber clay houses"

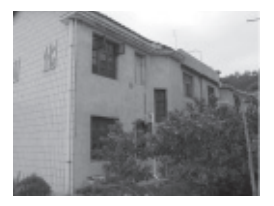

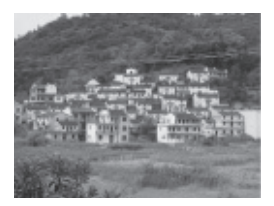

Photo 4. New YANG Village

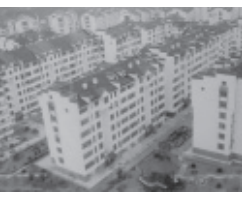

Photo 10. Urban Housing -ZIGUI, HUBEI Province

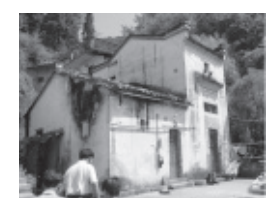

Photo 5. New YANG Village Photo 6. ZHANGTAN Village
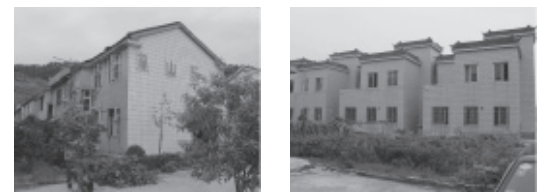

Photo 11. YINXINGTUO Village Photo 12. JIAJIA Village, ZIGUI, HUBEI Province -ZHEJIANG Province 


\begin{tabular}{|c|c|c|c|c|c|c|c|}
\hline \multicolumn{8}{|c|}{ Table 1. Site Investigation Data } \\
\hline $\bar{G}$ & $\mathrm{H}$ & I & $\mathrm{J}$ & $\mathrm{K}$ & $\mathrm{L}$ & $\mathrm{M}$ & \\
\hline \multicolumn{3}{|c|}{ HUBEI Province } & \multicolumn{4}{|c|}{ ZHEJIANG Province } & \\
\hline \multirow[b]{2}{*}{$\begin{array}{l}\text { YINXINGTUO Village, } \\
\text { ZIGUI }\end{array}$} & \multirow[b]{2}{*}{$\begin{array}{c}\text { JUYUAN Housing } \\
\text { Zone, } \\
\text { ZIGUI }\end{array}$} & \multirow{2}{*}{$\begin{array}{l}\text { GUIZHOU } \\
\text { Resettlement } \\
\text { Housing Zone, } \\
\text { ZIGUI }\end{array}$} & \multicolumn{2}{|c|}{ YUHANG Ward, HANGZHOU City } & \multicolumn{2}{|c|}{ WENZHOU City } & \\
\hline & & & $\begin{array}{c}\text { No.7 Group, } \\
\text { QIJIAQIAO Village, } \\
\text { YUNHE Town }\end{array}$ & $\begin{array}{l}\text { JIAJIA Village, } \\
\text { XINGQIAO Road }\end{array}$ & $\begin{array}{c}\text { CHENZHUANG } \\
\text { Village, } \\
\text { OUHAI Ward }\end{array}$ & $\begin{array}{l}\text { LONGHUA Village, } \\
\text { LONGWAN Ward }\end{array}$ & \\
\hline \multicolumn{3}{|c|}{ The State Council Three Gorges Project Construction Committee of China } & \multicolumn{2}{|c|}{$\begin{array}{l}\text { The State Council Three Gorges Project } \\
\text { Construction Committee of China }\end{array}$} & \multicolumn{2}{|c|}{ Resettlement Office of WENZHOU } & 1 \\
\hline \multicolumn{3}{|c|}{$\begin{array}{l}\text { THREE GORGES Dam/ } \\
1.2 \text { million }\end{array}$} & \multicolumn{2}{|c|}{$\begin{array}{l}\text { THREE GORGES Dam/ } \\
1.2 \text { million }\end{array}$} & \multicolumn{2}{|c|}{$\begin{array}{l}\text { SHANXI Dam } \\
45 \text { Thousand }\end{array}$} & 2 \\
\hline \multicolumn{3}{|c|}{$\begin{array}{l}\text { Central China/ } \\
\text { Average }\end{array}$} & \multicolumn{2}{|c|}{$\begin{array}{l}\text { Eastern China } \\
\text { Developed }\end{array}$} & \multicolumn{2}{|c|}{$\begin{array}{l}\text { Eastern China } \\
\text { Developed }\end{array}$} & 3 \\
\hline $\begin{array}{c}\text { Local-Rear- } \\
\text { Resettlement } \\
/ \text { - }\end{array}$ & $\begin{array}{c}\begin{array}{c}\text { Distance Resettlement } \\
\text { (Inside the County) } \\
/ 20 \mathrm{KM}\end{array} \\
\end{array}$ & \begin{tabular}{|c|} 
Distance Resettlement \\
(Inside the County) \\
/20KM
\end{tabular} & $\begin{array}{l}\text { Long Distance } \\
\text { Resettlement } \\
\text { /1500KM }\end{array}$ & $\begin{array}{c}\text { Long Distance } \\
\text { Resettlement } \\
/ 1500 \mathrm{KM}\end{array}$ & $\begin{array}{c}\begin{array}{c}\text { Distance Resettlement } \\
\text { (Inside the Province) } \\
/ 190 \mathrm{KM}\end{array} \\
\end{array}$ & \begin{tabular}{|c|} 
Distance Resettlement \\
(Inside the Province) \\
/220KM
\end{tabular} & 4 \\
\hline $1997.8-99.4$ & 1995-98 & $1999-2001$ & 2001.5.8 & 2001.5.8 & $1999.10-2000.11$ & $\begin{array}{c}1997.3-9 \\
1999.9-2000.5\end{array}$ & 5 \\
\hline $811 / 2580$ & $2190 / 7665$ & $168 / 588$ & $6 / 22$ & $4 / 17$ & $118 / 283$ & $844 / 2053$ & 6 \\
\hline Rural/Rural & Urban/Urban & Urban/Urban & Rural/Rural & Rural/Rural & Rural/Rural & Rural/Rural & 7 \\
\hline Yes/Yes & Yes/No & Yes/No & Yes/No & Yes/No & Yes/No & Yes/Yes & 8 \\
\hline Self Construction & Unifying Construction & Unifying Construction & Unifying Construction & Unifying Construction & Self Construction & $\begin{array}{l}\text { Cooperative } \\
\text { Construction }\end{array}$ & 9 \\
\hline Independent / Linear & \multicolumn{2}{|c|}{ Concentrated Housing / Concentrated Housing } & \multicolumn{2}{|c|}{ Independent Housing/Independent Housing } & \multicolumn{2}{|c|}{ Independent Housing/ Linear Housing } & 10 \\
\hline $\begin{array}{l}\text { Clay, Brick } \\
\text { /Brick+RC }\end{array}$ & $\begin{array}{l}\text { Brick, Brick+RC } \\
\text { /Brick+RC }\end{array}$ & $\begin{array}{l}\text { Brick, Brick+RC } \\
\text { /Brick+RC }\end{array}$ & $\begin{array}{l}\text { Clay, Brick } \\
\text { /Brick+RC }\end{array}$ & $\begin{array}{c}\text { Brick } \\
/ \text { Brick }+ \text { RC }\end{array}$ & $\begin{array}{l}\text { Clay, Brick } \\
\text { /Brick }+ \text { RC }\end{array}$ & $\begin{array}{l}\text { Clay, Brick } \\
\text { /Brick+RC }\end{array}$ & 11 \\
\hline 2001.1 .2 & $1-5 / 5-7$ & 2001.5 .3 & 2001.2 .2 & 2001.2 .2 & $1-2 / 3-3.5$ & 2001.2 .3 & 12 \\
\hline $1-3$ & $1-3$ & $1-3$ & $1-2$ & 2 & $1-2$ & $1-2$ & 13 \\
\hline 67.6 & 21.1 & 35.4 & 25 & 25 & 30 & 30 & 14 \\
\hline $35 / 45$ & $15 / 20$ & $20 / 32$ & $40 / 45$ & $25 / 30$ & $25 / 78$ & $32 / 69.5$ & 15 \\
\hline Whitewash, Tile & Tile & Tile, Paint & Tile & Tile & Tile & Tile & 16 \\
\hline $\begin{array}{c}\text { Tile } \\
\text { /Whitewash, Stucco }\end{array}$ & Ceramic tile/Paint & Ceramic tile/Paint & Ceramic tile/Paint & Ceramic tile/Paint & Ceramic tile/Paint & Ceramic tile/Paint & 17 \\
\hline \begin{tabular}{c|} 
Self-discharge Toilet \\
/Two-Urn-Funnel Toilet
\end{tabular} & $\begin{array}{c}\text { Flush toilet } \\
\text { /Flush toilet }\end{array}$ & $\begin{array}{l}\text { Flush toilet } \\
\text { /Flush toilet }\end{array}$ & $\begin{array}{l}\text { Self-discharge Toilet/ } \\
\text { Flush toilet }\end{array}$ & $\begin{array}{c}\text { Self-discharge Toilet/ } \\
\text { Flush toilet }\end{array}$ & $\begin{array}{c}\text { Self-discharge Toilet/ } \\
\text { Flush toilet }\end{array}$ & $\begin{array}{c}\text { Self-discharge Toilet/ } \\
\text { Flush toilet }\end{array}$ & 18 \\
\hline $\begin{array}{l}\text { Grocery, Dispensary, } \\
\text { Inn }\end{array}$ & \begin{tabular}{|c|} 
Shop, square, Parking, \\
Comfort station, \\
Hospital, Recreation \\
room
\end{tabular} & $\begin{array}{c}\text { Shop, square, Parking, } \\
\text { Comfort station, } \\
\text { Dispensary, Net-Bar }\end{array}$ & $\begin{array}{c}\text { Application of existing } \\
\text { neighbor- } \\
\text { hood public facilities }\end{array}$ & $\begin{array}{c}\text { Application of existing } \\
\text { neighbor- } \\
\text { hood public facilities }\end{array}$ & $\begin{array}{c}\text { Application of existing } \\
\text { neighbor- } \\
\text { hood public facilities }\end{array}$ & $\begin{array}{c}\text { Application of existing } \\
\text { neighbor- } \\
\text { hood public facilities }\end{array}$ & 19 \\
\hline Grade school & $\begin{array}{c}\text { Kindergarten, Grade } \\
\text { school }\end{array}$ & $\begin{array}{c}\text { Application of existing } \\
\text { neighbor-hood school }\end{array}$ & $\begin{array}{l}\text { Application of existing } \\
\text { neighbor-hood school }\end{array}$ & $\begin{array}{l}\text { Application of existing } \\
\text { neighbor-hood school }\end{array}$ & $\begin{array}{l}\text { Application of existing } \\
\text { neighbor-hood school }\end{array}$ & Grade school & 20 \\
\hline $1-5 \mathrm{Km}$ & $0-2 \mathrm{Km}$ & $0-3 \mathrm{Km}$ & $0-2 \mathrm{Km}$ & $0-2 \mathrm{Km}$ & $1-5 \mathrm{Km}$ & $1-5 \mathrm{Km}$ & 21 \\
\hline \begin{tabular}{c|}
$\begin{array}{c}\text { Application of existing } \\
\text { main road }\end{array}$ \\
\end{tabular} & Yes $/ 7 \mathrm{~m}, 5 \mathrm{~m}, 3.5 \mathrm{~m}$ & Sidewalk & Yes $/ 3.5 \mathrm{~m}$ & $\mathrm{Yes} / 3.5 \mathrm{~m}$ & Yes $/ 5 \mathrm{~m}$ & $\mathrm{Yes} / 5 \mathrm{~m}$ & 22 \\
\hline $\begin{array}{l}\text { Bike, Motorcycle, } \\
\text { Farming-vehicle/ } \\
\text { convenient }\end{array}$ & \multicolumn{2}{|c|}{$\begin{array}{l}\text { Bike, Motorcycle, } \\
\text { Car/ convenient }\end{array}$} & $\begin{array}{l}\text { Bike, Motorcycle } \\
\text { / convenient }\end{array}$ & $\begin{array}{l}\text { Bike, Motorcycle } \\
\text { / convenient }\end{array}$ & $\begin{array}{l}\text { Bike, Motorcycle, } \\
\text { Car / convenient }\end{array}$ & $\begin{array}{l}\text { Bike, Motorcycle, } \\
\text { Car/ convenient }\end{array}$ & 23 \\
\hline $\begin{array}{l}\text { Farming/Farming, } \\
\text { Trade, Service }\end{array}$ & \multicolumn{2}{|c|}{$\begin{array}{l}\text { Industrial, Trade, Administrative } \\
\text { /Industrial, Trade, Administrative }\end{array}$} & $\begin{array}{l}\text { Farming/Farming, } \\
\text { Trade, Service }\end{array}$ & $\begin{array}{l}\text { Farming/Farming, } \\
\text { Trade, Service }\end{array}$ & \multicolumn{2}{|c|}{ Farming/Farming, Trade, Service, Industrial } & 24 \\
\hline No & $\begin{array}{l}\text { Compatible with } \\
\text { Commercial Growth }\end{array}$ & $\begin{array}{c}1 \text { st Floor for } \\
\text { Commercial and } \\
\text { Service Spaces }\end{array}$ & No & No & No & No & 25 \\
\hline G & $\mathrm{H}$ & I & $\mathrm{J}$ & $\mathrm{K}$ & $\mathrm{L}$ & $\mathrm{M}$ & \\
\hline
\end{tabular}

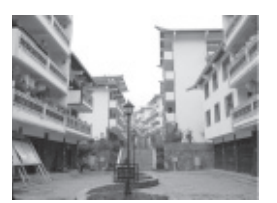

Photo 13. GUIZHOU Resettlement Photo 14. MAYU Village, Housing Zone (Unified Construction)

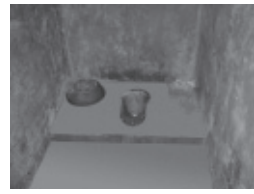
Photo 18. Two-Urn-Funnel Photo 19. Flush toilet

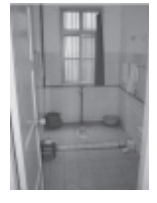

Pho

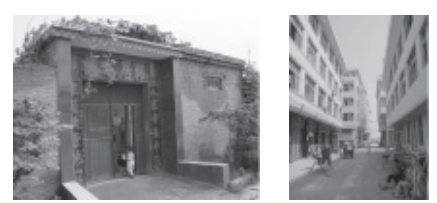

Photo 15. CHENZHUANG Village, ZHEJIANG (Cooperative Construction)

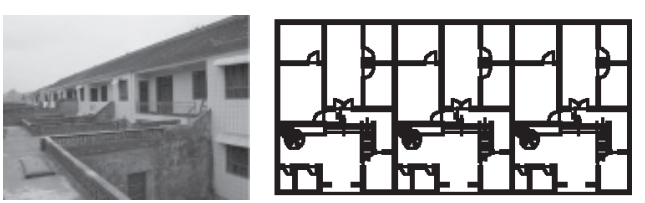

Photo 16. and Fig.6. Linear Housing, ZAILI Village,

HUANQU, SHANXI Province

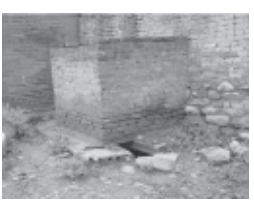

Photo 17. Self-discharge Toilet

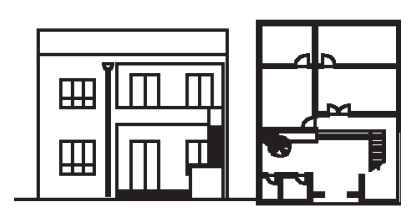

Figs. 2 and 3 "Typical Design",
ZAILI Village, HUANQU, SHANXI

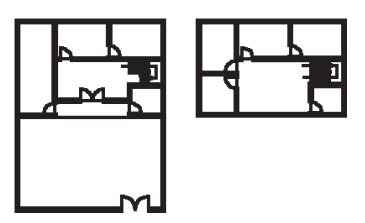

Fig.4. “Typical Design” CANGTOU Village WENXIAN, HENAN $(1,2 \mathrm{~F})$
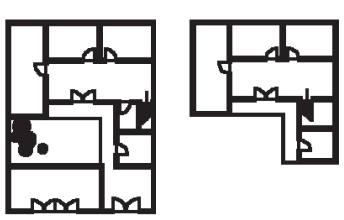

Fig.5. A Change of "Typical Design", CANGTOU Village, WENXIAN, HENAN
Planned Economy the difference in economic conditions was by and large similar everywhere in China. After 20 years of the application of "Reform and Opening" policies the Eastern region greatly surpasses the Western region in terms of economics.
Yet Chinese policy regarding dam resettlement was carried out based on consistent guidance and bylaws by the Central Governmental. This set of bylaws was issued to local governments and allowed local officials to adjust them to meet their own needs. As 
a result housing construction in many regions differs in terms of choice of land, average dwelling area per person, construction materials and structural styles and decoration. Such diversity has a negative psychological impact on resettlers.

\subsection{Resettlement Housing Policy and Construction: Urban vs. Rural}

In this author's investigation, urban resettlement housing differs from that in ruban areas in that $95 \%$ of the former resettlers live in concentrated housing with $25 \mathrm{~m}^{2}$ per person, whereas the rural resettlers live in single units with $45 \mathrm{~m}^{2}$ per person. These figures seem to favor the rural resettlers; yet in terms of construction quality, materials, equipment and hygiene facilities the urban units are superior (Table 1. 7, 10-23/G-I) (Photos 9 and 10).

Urban and rural housing have their own bylaws concerning principles of housing construction.

The urban housing units were carried out by consignment; that is, urban resettlers relegate, through their business enterprise, the housing construction to the Bureau of Resettlement, which implements a unified management principle for the construction of housing units. This principle proved effective in dealing with limited urban building areas, high-cost materials, and large quantity of units and technical difficulties due to complicated infrastructure. Thus, concentrated housing, unified design, unified construction and unified management becomes the principle for urban resettlement housing.

Rural housing units are implemented in a selfsufficient manner. This is due in part to the fact that rural resettlers are farmers who do not belong to any institute and thus are hard to manage. Owing to the flexible building areas, less-complicated structure, low-cost materials and construction, rural resettlement housing has the following characteristics: individual units, unified design, self-supported construction and the combined efforts of several families.

"Agriculture Resettlement" has been the primary concern for the Chinese government in the resettlement scenario; i.e., those who were farming should farm in their resettlement area. But the reality was that the new settlers turned to other vocations like, handmanufacturing, trade, the service business, and so on. Thus, resettlement-housing design needs to be adjusted to suit such change. The current housing designs however, fail to produce corresponding schemes to accommodate change and allow the resettlers to renovate their chosen units in accordance with their needs. (Table 1. 8, 24, 25) (Figs. 4 and 5)

\subsection{Construction Diversities: "Distance}

\section{Resettlement" ${ }^{4}$ ) vs. "Local-Rear-Resettlement" ${ }^{5}$ )}

Chinese Dam Resettlement has been carried out in two ways: "Distance Resettlement" and "Local-RearResettlement."

From the author's investigation, each method of resettlement has its own housing construction style.

Local-Rear-Resettlement is more adaptable than Distance resettlement. By the former, resettlers already used to their living environment and resettlement housing in general could adapt to this style. The resettlement regions were planned and built by employing the pattern of the old village housing units. Therefore, the new resettlement village would be by and large similar to the old one in housing style, villager composition and neighborhood relations.

The long distance resettlers, having spent time and energy moving, are unable to spend time rebuilding their ideal housing units. They need to move in and settle after a long journey. Thus the housing policy should be altered and characterized by unifying both the design of individual units as well as construction and management, and moving quickly. This guidance turned out to be the principle for long distance resettlers, which, compared with those of the local rear resettlement, was closer to the urban resettlement housing construction. (Table 1. 4, 9/G, J, K)

The current on-going "Three Gorges Project" is the largest dam construction in Chinese History. The resettlement population has reached 1,130,000, of which some 125,000 — the largest long distance resettlement in history — was carried out with lessons learned from previous resettlements; the resettler's personal interests were respected. The long distance resettlers received compensation not only from the central government but also from the local government. This "three-gorges" long distance resettlement case was far better than the cases of local-rear-resettlement with respect to housing unit area, construction and decoration materials, hygiene facilities and so on (Table1. 4, 10-23/G, J, K) (Photos 11 and 12).

Yet, in terms of the unity of the whole resettled hamlet, long distance resettlement has several problems. People, mostly farmers of the same village were separated in the name of the so-called "scattering resettlement" and were moved to different resettlement villages. The purpose was to encourage the new settlers to merge with the local residents and become accustomed to their new environment. However, an interview shows that most resettlers feel resistant to the new social relationship and are unable to adapt to the new neighborhood because of the local vernacular and customs. Thus they tend to group themselves in a set of families consisting of four or six units. The result is a psychological burden, a social symptom - known as the so-called "vulnerable social group."

\subsection{Construction Styles of Resettlement Housing}

The author's investigation found that resettlementhousing construction was not uniformly carried out via government but by the means of various sources. Why? Resettlement policies, lacking a set of resettlement housing policies, allow the local administration to do so in accordance with local needs. Housing 
construction for the Chinese dam resettlement basically consists of the following three methods.

\subsubsection{Unified Construction by Government}

This method is sometimes called "Consignment Construction," referring to a contract signed by representatives from government and the resettlement group. The government side shoulders the responsibilities for a unified housing unit scheme, design, construction, finishing and delivery to the resettlers. The resettlers are never involved in the whole process, not to mention changing the housing design. This method is largely employed by urban and long-distance resettlement (Photo 13).

Merits: schemes and designs are under a unified management. Good housing quality with sets of appliances. Short construction period and unified occupancy.

Disadvantages: without the resettler's participation in supplying information to make the designs on behalf of the resettler's immediate needs and future development, disagreements between settlers and government do occur: unreasonable designs, partial construction defects and selfish excessive-requests by the resettlers themselves.

\subsubsection{Self Construction by Resettlers}

In this method the government offers land and at least three housing design proposals — "typical designs" - for the resettlers. The resettlers and the village jointly select one; the settlers either build it themselves or assign a sub-contractor to do the job. The former self-built process packaged tasks including the purchase of material and various phases of construction. The latter, with a contractor, let the resettler observe and even be involved in the whole construction process; small changes from the blueprints required by the settlers to meet their needs are permitted. In a scenario like this, several families usually unite and select among themselves a representative to sign the contract and supervise the construction process. The main stream of the resettlement, mostly agricultural workers, is Local-Rear-Resettlement, in which $90 \%$ of settlers self-construct their housing units (Photo 14).

Merits: The chance to change the blueprints of the "typical Design", and their involvement and supervision during the construction process minimizes disagreement between the government and themselves. The occupation follows smoothly.

Disadvantages: Owing to a lack of professionals to supervise the construction, the quality is weak. Changing from the blueprints of the "typical design" without advice from professionals surely jeopardizes the structural safety and residential rationality.

3.4.3 Cooperative Construction

In this method the local government is the primary party for the construction while representatives from the settlers are invited to observe the construction process. The aim is to reduce arguments regarding the quality of the finished housing units, and is also about the transparency level of the local government. This method has not been widely implemented except in a few economically well-off regions with open-minded governments. (Photo 15).

Merits: The quality of construction is more guaranteed than the previous method, due to cooperation between the government and the settlers. Since the construction process is open, settler representatives are allowed to participate in various ways, thereby reducing disagreement. In theory this method of dam resettlement housing construction is the most reasonable one.

Disadvantages: This method places high demands on both the government and the resettlers. It requires greater transparency from government for the settlers to realize the construction with respect to economics, site working procedures and engineering. It requires a high educational level from the settlers so that their requests for change concerning materials, construction processes or even blueprints in the "typical designs" are reasonable. Yet quite a few local governments and settlers still need to upgrade their own qualities not only professionally but personally as well. It is not easy to make this method widely popular.

\section{Conclusion}

The Chinese Dam Resettlement Housing Construction has been complicated, involving aspects of economic orientation, political climate, technique and humanity. Through this site investigation the author is trying to study resettlement housing against the backdrop of the Chinese system of tradition, policy, regional diversity, planning, government methodology and the vicissitudes of resettlement housing evaluation standards.

1) Chinese dam resettlement was compulsory in its early stage, then semi-compulsory all the way up to the current situation in which the rights of resettlers are respected and resettlement policies legalized. Housing construction dramatically changed from the early shelter-like units to the current ones which are even better than the average Chinese resettlement housing units today in many regards such as larger living area, better quality construction and hygienic level.

2) Without a self-system, the resettlement housing policy was not independent; it consisted of a set of vague bylaws as a sub-branch under the Grand Resettlement Policy. In the early stage, due to the weak economic situation and poor production capability, the concern of how to provide for the resettlers always surpassed how to house them comfortably. After the 1980 's, with the growth of economics and production capabilities, the resettlers then turned to the pursuit of housing quality. Corresponding to this change, an independent housing policy is a must. 
3) Regional diversity distinguishes the East and West of China, as well as the urban and rural areas. A prosperous housing unit in the East enjoys on average a larger area 1.5 to 2 times that in the West. Construction quality, hygiene and so on also differ. Housing units in the rural areas are larger than those in the urban areas; yet in other aspects may be a lot worse.

4) The resettlement-housing scheme does not correspond to the change of life style of the resettlers. That the Chinese government, following the principle that "Agriculture is Primary," expects a farmer to carry on farming in the new resettlement is understandable, however, the reality is that the resettlers have to adjust in accordance with rapid change of the economic climate. In other words, the farming-oriented housing design does not suit the resettler's practical needs. Thus, the resettlers reform the "typical design" to suit their needs. Considering the fact that China is at a turning point in terms of prosperity, resettlementhousing design for tomorrow is an urgent issue.

5) During the process of housing construction the government should adopt an open attitude towards housing policy, in order to win the trust of resettlers. Self-constructed housing by the resettlers may have merits that favor them yet these units do have problems in terms of quality and safety. Thus, trust between the government and the resettlers in building the housing units is a future issue regarding the cooperative construction of resettlement housing.

6) Evaluation of resettlement housing in the period from the birth of the PRC until the 1970's was simple due to the low dwelling standard; basically it was judged by the data of the average living area per person, and this method of evaluation is still used today. Since the 1980's both urban and rural resettlers began to enjoy a higher standard of living and demanded a higher level of housing quality. Issues such as reasonable living space, structural styles of housing, hygienic environment, housing facilities and decoration, appliances and the distance between home and production area, effective and convenient transportation and so on are taken into account in terms of new evaluation standards.
This research, together with the site investigation, was carried out with a broad point of view regarding the history and characteristics of the Chinese Dam Resettlement Housing Construction, and it is hoped that further research concerning the specific conditions, living standards and housing styles will be continued.

\section{Endnotes}

1) The Great Leap Forward, which occurred in the People's Republic of China from 1958 to early 1960 aimed at using mainland China's abundant supply of labor to rapidly industrialize the nation

2) The Cultural Revolution was a political movement in the People's Republic of China, which lasted from (1966-1976). The economic system, traditional culture and sense of values were severely damaged.

3) Based on Information of the Ministry of Construction of the PRC, the national average living area per person is; Urban, $9.3 \mathrm{~m} 2$ and Rural, $23.7 \mathrm{~m} 2$.

4) Distance Resettlement refers to the resettlement method carried out while compromising environmental restrictions and the natural environment. Thus, the resettlers have no choice but to move elsewhere. Moving distances range from $50 \mathrm{~km}$ to $2,000 \mathrm{~km}$. In general, resettling without moving outside of their administrative region or moving within $100 \mathrm{~km}$ is termed Distant Resettlement, while moving over $100 \mathrm{~km}$ to regions of different administration or racial group is termed "Long Distance Resettlement."

5) Local-Rear-Resettlement refers to the resettlement method which moved the resettlers to a higher elevation of their original residential area to avoid dam water.

6) Similar to the "NAGAYA" in Japan, the Concentrated Housing is set in rows with 3-6 single units.

7) Self-discharge toilet — aging toilets with the lowest hygienic condition without water and sterilization appliances.

8) A $1 \mathrm{~m} 3$ container attached to the toilet bowl for sterilization and sedimentation, plus an extra same-size container attached to deposit the sterilized waste for agricultural purposes. This kind of toilet has a better hygiene level than the self-discharge toilet.

\section{References}

1) Shenyi, AN et al. (2000) Hydropower Engineering in China Part of Resettlement and Environmental Protection, China Electric Power Press.

2) Michael M. CERNEA (1998) Studies of World Bank Resettlement Policies And Experience II - Resettlement, Rehabilitation, Development, HOHAI University Press, 1998

3) Hengshun, LEI et al. (2002) The Three Gorges Immigration of China, CHONGQING University Press

4) Daoshu PANG (2001) A Guide to Dam-Resettlement, HUNAN Publishing House.

5) Guoqing SHI et al. (2001) China Resettlement Policy and Practice 